Review Article

\title{
Baicalein, Baicalin, and Wogonin: Protective Effects against Ischemia-Induced Neurodegeneration in the Brain and Retina
}

\author{
Li Pan $\mathbb{D}^{1,2}$ Kin-Sang Cho $\mathbb{D}^{2},^{2}$ Irvin Yi $\mathbb{D},^{2}$ Chi-Ho To $\mathbb{D},,^{1,3}$ Dong Feng Chen $\mathbb{D},^{2}$ \\ and Chi-Wai Do $\mathbb{D}^{1,3}$ \\ ${ }^{1}$ School of Optometry, The Hong Kong Polytechnic University, Hong Kong \\ ${ }^{2}$ Schepens Eye Research Institute of Massachusetts Eye and Ear, Department of Ophthalmology, Harvard Medical School, Boston, \\ MA, USA \\ ${ }^{3}$ Centre for Eye and Vision Research, Hong Kong
}

Correspondence should be addressed to Dong Feng Chen; dongfeng_chen@meei.harvard.edu and Chi-Wai Do; chi-wai.do@polyu.edu.hk

Received 30 April 2021; Revised 8 June 2021; Accepted 19 June 2021; Published 30 June 2021

Academic Editor: Juan Gambini

Copyright ( $92021 \mathrm{Li}$ Pan et al. This is an open access article distributed under the Creative Commons Attribution License, which permits unrestricted use, distribution, and reproduction in any medium, provided the original work is properly cited.

\begin{abstract}
Ischemia is a common pathological condition present in many neurodegenerative diseases, including ischemic stroke, retinal vascular occlusion, diabetic retinopathy, and glaucoma, threatening the sight and lives of millions of people globally. Ischemia can trigger excessive oxidative stress, inflammation, and vascular dysfunction, leading to the disruption of tissue homeostasis and, ultimately, cell death. Current therapies are very limited and have a narrow time window for effective treatment. Thus, there is an urgent need to develop more effective therapeutic options for ischemia-induced neural injuries. With emerging reports on the pharmacological properties of natural flavonoids, these compounds present potent antioxidative, antiinflammatory, and antiapoptotic agents for the treatment of ischemic insults. Three major active flavonoids, baicalein, baicalin, and wogonin, have been extracted from Scutellaria baicalensis Georgi (S. baicalensis); all of which are reported to have low cytotoxicity. They have been demonstrated to exert promising pharmacological capabilities in preventing cell and tissue damage. This review focuses on the therapeutic potentials of these flavonoids against ischemia-induced neurotoxicity and damage in the brain and retina. The bioactivity and bioavailability of baicalein, baicalin, and wogonin are also discussed. It is with hope that the therapeutic potential of these flavonoids can be utilized and developed as natural treatments for ischemia-induced injuries of the central nervous system (CNS).
\end{abstract}

\section{Introduction}

Ischemia is a common pathological or traumatic condition accompanied by the reduction of blood supply to the major organs, such as the heart, kidney, intestine, brain, and eye [1]. This leads to an insufficient supply of oxygen and nutrients and an accumulation of metabolic wastes, causing organ damage or failure and resulting in death in severe cases [1]. Neurons in the brain are the most sensitive and vulnerable cells to ischemia. Only a short period of ischemia can elicit irreversible damage to brain tissue, leading to paralysis or death $[2,3]$. Stroke was defined by the World Health Organization (WHO) in the 1970s as "rapidly developing clinical signs of focal disturbance of cere- bral function, lasting more than 24 hours or leading to death with no apparent cause other than that of vascular origin" [4]. Around $87 \%$ of stroke cases are ischemic stroke, which is triggered by a lack of blood supply to focal brain areas, leading to subsequent damage and neurodegeneration [5-7]. Stroke is a leading cause of disability and death worldwide $[7,8]$. Thrombolytic medication, such as alteplase (t-PA), is the only FDA-approved therapeutic agent for treating acute ischemic stroke within a few hours after its onset [9]. Given the narrow time window of treatment and high risk of complications, such as hemorrhagic transformation, cerebral edema, and other adverse effects [10], the development of novel neuroprotective therapies against ischemia is paramount. 
The visual system is comprised of the sensory organ (eyes) and connecting axon fibers to the visual targets of the brain [11]. Light, as a stimulus, is captured by photoreceptors in the retina, initiating a cascade of chemical and electrical events. The signal is then transferred to the visual center of the brain via the ganglion cell axons of the optic nerve [12-14]. The visual centers process and transform these signals into visual images. Retinal ischemia is frequently involved in various forms of retinal neuropathies, such as age-related macular degeneration (AMD), diabetic retinopathy (DR), glaucoma, and central/branch retinal artery/vein occlusion [15-19]. Following ischemic injuries, a series of events are triggered, including oxidative stress, neovascular and apoptotic changes, and, ultimately, the death of retinal neurons and vision loss $[5,20]$. The retina is an extension of the brain in terms of anatomical and embryonic development [21, 22]. The retina also displays similarities to the brain regarding its neuronal and immune responses to injury [22]. The latter is possibly contributed by the structural similarity between the blood-retinal barrier (BRB) and blood-brain barrier (BBB), to which the retina sustains an immune privilege site and shares a similar pattern of immune surveillance and immunoregulatory processes $[23,24]$. In response to perturbations in the retina and the brain, innate immunity can be rapidly activated through transcriptional and phenotypic alterations of immune glial cells and the release of inflammatory cytokines $[25,26]$. However, excessive activation of innate immune reactivity under injury or traumatic stress can promote further activation of adaptive immunity by antigen-presenting cells that attract and guide peripheral immune cells, such as $\mathrm{T}$ cells to the injury sites [27-29]. Neuroinflammation has been well documented as a pathological factor in neuronal death in the brain and in retinal disorders. Because of these similarities, the retina has been commonly considered as an easily accessible indicator of brain disorders, such as Alzheimer's disease (AD), Parkinson's disease (PD), and stroke.

Accumulating evidence suggests that natural herbs exhibit therapeutic potential for the treatment of ischemic stroke [30]. Active ingredients extracted from herbs, including salvianolic acid B and tanshinone from Salvia miltiorrhiza, scutellarin from Scutellaria baicalensis Georgi ( $S$. baicalensis), and honokiol and magnolol from the bark of Magnolia officinalis, have been found to have therapeutic potential, because of their antioxidative and antiinflammatory properties, as well as their ability to maintain BBB permeability [30-36]. The neuroprotective capabilities of other natural extracts, including resveratrol, curcumin, vitamins $\mathrm{C}$ and $\mathrm{E}$, and Gingko biloba, have also been reported in various CNS disorders [37]. Flavonoids, which are easily accessible by daily consumption of fruits and vegetables, have been found to have high therapeutic efficacy and fewer side effects in both in vitro and in vivo studies [38, 39]. Because of their various pharmacological effects, many flavonoids have demonstrated promising protective effects in the prevention or treatment of various diseases [40-46]. This review mainly focuses on three flavonoids: baicalein $(5,6,7-$ trihydroxyflavone; $\mathrm{C}_{15} \mathrm{H}_{10} \mathrm{O}_{5}$ ), baicalin (5,6-dihydroxy-7-Oglucuronide), and wogonin (5,7-dihydroxy-8-methoxy-fla- vone) (Figure 1), all of which are isolated from the roots of S. baicalensis, a widely used herbal medicine in Asian countries [47-51]. Previous studies have demonstrated that baicalein, baicalin, and wogonin have a broad spectrum of biological functions, including antioxidation, anti-inflammation, antiapoptosis, and antiexcitotoxicity $[51,52]$. Because of these bioactivities, many published reports have suggested the possibility of developing flavonoids for the treatment of various diseases, including hepatitis, breast cancer, virus infection, and neurodegenerative diseases [43-46]. These pharmacological activities also provide a solid basis for their neuroprotective properties in different models of neuropathies and cognitive impairments. Because of their easy accessibility and low toxicity, baicalein, baicalin, and wogonin may be effective alternatives for the treatment of stroke and other neurodegenerative diseases affecting CNS.

\section{Bioactivity and Safety of Baicalein, Baicalin, and Wogonin}

As the major active flavonoids extracted from S. baicalensis, baicalein, baicalin, and wogonin share some common pharmacological properties against inflammation, oxidation, and apoptosis. A comparison of baicalein, baicalin, and wogonin is shown in Table 1, based on the parameters collected from the database of the traditional Chinese medicine lab of systems pharmacology (TCMSP), a database integrating systems biology and pharmacology for drug discovery, development, and understanding of therapeutic mechanisms [53]. The database can be found in the following link (https:// old.tcmsp-e.com/tcmsp.php).

Baicalein and wogonin have a lower molecular weight (MW) and a lower value of the topological polar surface area (TPSA) than baicalin, indicating a higher cell membrane permeability of baicalein and wogonin [54]. Furthermore, the permeability of Caco-2 monolayers (intestinal epithelial cells) and the BBB, calculated based on the values of TPSA $[55,56]$, was found to be higher for baicalein and wogonin, compared to baicalin. In addition, baicalein and wogonin display slower elimination half-time (HL) and higher oral bioavailability $(\mathrm{OB})$ compared to baicalin, suggesting a longer duration of these two flavonoids in systemic circulation. Based on multiparametric guidelines, also known as rules and ligand efficiency (LE) metrics, which determine the extent of druglikeness (DL) [57], all three flavonoids meet the criteria of "drug-like" compounds. This demonstrates the potential of baicalein, baicalin, and wogonin to be easily accessible agents for future clinical use. However, the relatively high hydrophobicity of baicalein and wogonin is reflected by their lipophilicity (AlogP, a logarithm of 1-octanol/water partition coefficient) values [58], compared to that of baicalin. Due to this low water solubility, solvents or carriers may be necessary to enhance the solubility of these flavonoids for therapeutic purposes [50, 59-61].

$S$. baicalensis is a major ingredient in many prescriptions of traditional Chinese medicine (TCM). Numerous studies have been conducted to evaluate its pharmacokinetic profile and bioavailability for its safety and efficacy in clinical applications. It has been reported that baicalein and its metabolite 


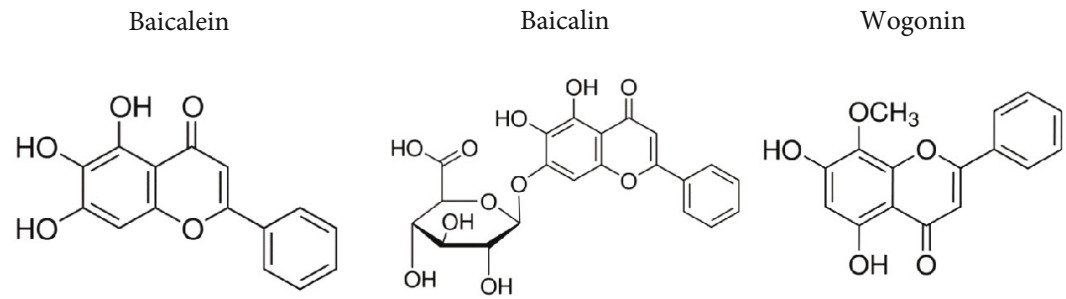

Figure 1: Molecular structures of baicalein, baicalin, and wogonin.

TABLE 1: Summary of physical parameters of baicalein, baicalin, and wogonin. MW: molecular weight (Dalton (Da)); TPSA: topological polar surface area (angstroms squared $\left(\mathrm{A}^{2}\right)$ ) indicates the membrane permeability; Caco-2: Caco-2 cell monolayer permeability $\left(10^{-6} \mathrm{~cm} \cdot \mathrm{s}^{-1}\right)$; and BBB: blood-brain barrier permeability $\left(10^{-6} \mathrm{~cm} \cdot \mathrm{s}^{-1}\right)$ were derived based on TPSA; half-time (HL) (hours (h)), oral bioavailability (OB) (\%), the logarithm of 1-octanol/water partition coefficient $(\mathrm{AlogP})$, and drug-likeness (DL) represent the pharmacological properties for each molecule.

\begin{tabular}{lcccccccc}
\hline Molecule & MW $(\mathrm{Da})$ & TPSA $\left(\mathrm{A}^{2}\right)$ & $\mathrm{Caco}-2\left(10^{-6} \mathrm{~cm} \cdot \mathrm{s}^{-1}\right)$ & $\mathrm{BBB}\left(10^{-6} \mathrm{~cm} \cdot \mathrm{s}^{-1}\right)$ & HL $(\mathrm{h})$ & OB $(\%)$ & AlogP & DL \\
\hline Baicalein & 270.25 & 90.9 & 0.63 & -0.05 & 16.25 & 33.52 & 2.33 & 0.21 \\
Baicalin & 460.42 & 187.12 & -1.1 & -1.97 & - & 29.53 & 0.84 & 0.77 \\
Wogonin & 284.28 & 79.9 & 0.79 & 0.04 & 17.75 & 30.68 & 2.56 & 0.23 \\
\hline
\end{tabular}

baicalein 6-O-sulfate exist in blood plasma for up to 36 hours after a single oral administration of Xiaochaihu Tang (ShoSaiko-To) [62], a popular TCM treatment containing extract of S. baicalensis. The bioavailability of wogonin, baicalein, and baicalin has also been evaluated in healthy human urine. After a single administration of $S$. baicalensis decoction (equal to $9 \mathrm{~g}$ of crude drug), wogonin, baicalein, and baicalin were still detectable in the urine 36 hours postdosing [63]. A similar time profile has also been demonstrated in monkey plasma after three doses of baicalein [64]. The presence of baicalin has been detected in human plasma after administration $[65,66]$. Additionally, the safety profile of single or multiple administrations of chewable baicalein tablets has been assessed in healthy subjects. In addition to detecting sustained levels of baicalein and its metabolite baicalin in vivo, these studies revealed that single or multiple doses of baicalein (100-800 mg) were safe and well tolerated with no sign of toxicity in the kidney or liver $[67,68]$. These findings implicate the feasibility of developing baicalein, baicalin, and wogonin as safe and long-lasting agents for clinical application.

\section{Neuroprotective Effects of Baicalein, Baicalin, and Wogonin on the Brain and Retina Ischemia}

Neurodegeneration often occurs through the progressive loss of the structure or functions of neurons [69]. Neurodegenerative diseases, such as Parkinson's disease (PD), Alzheimer's disease (AD), and stroke, affect millions of people worldwide, especially in aging populations. The pathogenesis of neurodegeneration is complicated and has been associated with genetics, protein misfolding, intracellular mechanisms, and programmed cell death [70-74]. In ischemic stroke patients, typical symptoms are characterized by the sudden loss of mobility, speaking, or vision unilaterally [75]. Ischemia in the brain can be caused by cardioembolic vessel occlusion, artery to artery embolism, or in-situ small-vessel disease [75]. In the retina, ischemia can result from occlusion of the central or branch retinal vessels or DR [76]. Retinal ischemia may lead to visual impairment and blindness [19].

Ischemia induces a cascade of neuropathological activities, including oxygen and energy depletion, disruption of ion homoeostasis, glutamate and free radical release, $\mathrm{Ca}^{2+}$ channel dysfunction, BBB or BRB disruption, and changes to the inflammatory microenvironment, ultimately leading to cell death and irreversible functional loss $[5,77,78]$. The cellular changes after ischemic stroke are illustrated in Figure 2. There are three major types of stress. First, oxidative stress is induced by rapidly increased reactive oxygen species (ROS) postischemia [79]. This stress can subsequently lead to the peroxidation of membrane lipid, mitochondrial dysfunction, and DNA damage, eventually causing apoptosis and irreversible neuron loss [80, 81]. Second, neuroinflammatory stress, initiated by the activation of innate and adaptive immunity, is a well-known pathological factor in CNS disorders $[82,83]$. The resident immune cells (microglia and astrocytes) can be rapidly activated upon sensing damageassociated molecular patterns (DAMPs) released by apoptotic cells $[83,84]$. Subsequently, adaptive immune cells can be recruited even with minimized disturbances or disruptions of $\mathrm{BBB}$ or $\mathrm{BRB}$ postischemia insults, ultimately causing neuron death $[29,69,85]$. Lastly, stress of energy deprivation triggers cytotoxicity at the lesion site. Apart from the excessive free radicals, the disruption of ion homeostasis postischemia/reperfusion injury can lead to overload of $\mathrm{Na}^{+}$ and $\mathrm{Ca}^{2+}$, which aggravates mitochondrial dysfunction and initiates apoptosis and inflammatory cascades [86]. Furthermore, the release and accumulation of glutamate can cause excitotoxicity of neurons and ultimately leads to cell death $[87,88]$. Based on the understanding of neurochemical events under ischemic stress, many neuroprotective therapies are focused on targeting the upstream pathways to reduce 


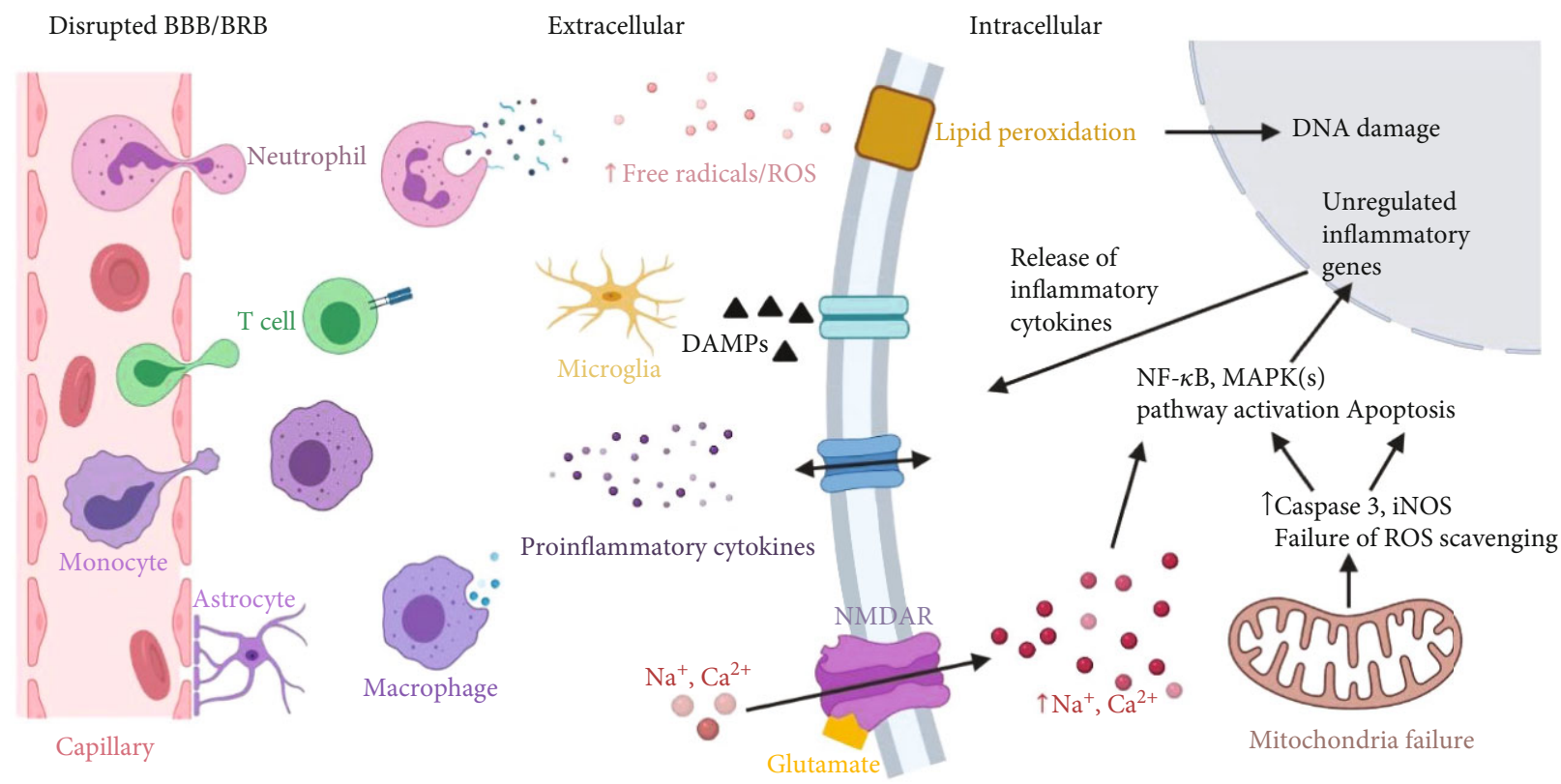

FIGURE 2: Schematic representation of the main pathological events subsequent to brain ischemia. Created with http://BioRender.com.

damage to the neurons induced by downstream cascades. The protective effects of natural herbs have been demonstrated by many research groups [89]. In the following discussion, the neuroprotective effects of baicalein, baicalin, and wogonin, the major bioactive molecules in S. baicalensis, are described based on the sites (i.e., the brain and retina) of postischemic injury.

3.1. Ischemia in the Brain. Following ischemic injury in the brain, a cascade of changes is elicited, including oxygen and energy deprivation, increased expression of free radicals and inflammatory cytokines, ion overload, and activation of immune responses [90,91]. The upstream triggers of these changes and maintenance of tissue homeostasis are recognized as potential therapeutic targets for ischemic stroke treatment. Multiple pharmacological properties of flavonoids as reported in the literature include antioxidant, anti-inflammation, antiapoptosis, and antiexcitotoxicity characteristics that are neuroprotective $[92,93]$. The neuroprotective effects of baicalein, baicalin, and wogonin on both in vitro and in vivo ischemic models are discussed (also see Tables 2 and 3 for details).

3.1.1. In Vitro Studies. Free radicals and ROS are known as inducers of cell death postischemic injury. Baicalein has been shown to exert neuroprotective effects and attenuate apoptosis by acting as a scavenger of ROS and nitric oxide (NO) [94]. These baicalein-induced protective effects have been found to be mediated by upregulating the phosphatase and tensin homolog gene (PTEN) and the phosphoinositide 3kinase (PI3K/AKT) pathway in primary cultures of cortical neurons after oxygen and glucose deprivation (OGD) treatment [94]. Additionally, it has been reported that baicalein improves the survival of HT22 cells after iodoacetic acid(IAA-) induced oxidative stress, by inhibiting 12/15-lipoxy- genase (12/15-LOX) [93], an enzyme which oxidizes polyunsaturated fatty acids to generate bioactive lipid metabolites [95] and is toxic to neurons in neurological disorders [96].

Neuroprotective abilities of wogonin and baicalin have also been reported. In OGD-induced toxicity to rat hippocampal slice culture, pretreatment and posttreatment of hippocampal slices with $50 \mu \mathrm{M}$ baicalin could significantly prevent cell death, especially in the pyramidal cell layer [97]. Wogonin has been demonstrated to act as an antioxidant and 12/15-LOX inhibitor to improve the survival of primary cortical neurons after $\mathrm{H}_{2} \mathrm{O}_{2}$ or xanthine/xanthine oxidase challenge [98].

It is worth noting that not only oxidative stress but also the dysregulated immune microenvironment can lead to the degeneration of brain cells. In OGD-induced ischemia of PC12 cells, which were derived from a pheochromocytoma of the rat adrenal medulla, baicalein, baicalin, and wogonin all exhibited significant neuroprotective effects by inhibiting oxidation and suppressing inflammation [99]. These protective effects were shown to be mediated by the reduced expression of Toll-like receptor 2 (TLR2), tumor necrosis factor alpha (TNF- $\alpha$ ), and caspase-3 [99]. Baicalin acts through suppressing OGD-induced phosphorylation of calmodulin- (CaM-) dependent protein kinase II (CaMKII) in primary hippocampal neurons and SH-SY5Y cells [100]. The antiapoptotic effects of baicalin have also been shown to suppress caspase- 3 and Bax expression and to promote antiapoptotic factor Bcl-2 expression in hippocampal neurons [100]. The effect of baicalin on rescuing neurons from OGD was comparable with that of CaMKII siRNA knockdown in SH-SY5Y cells, suggesting that baicalin may function as a potent CaMKII inhibitor in neuroprotection [100].

3.1.2. In Vivo Studies. Neuroprotective effects of baicalein, baicalin, and wogonin against ischemic injuries have been 


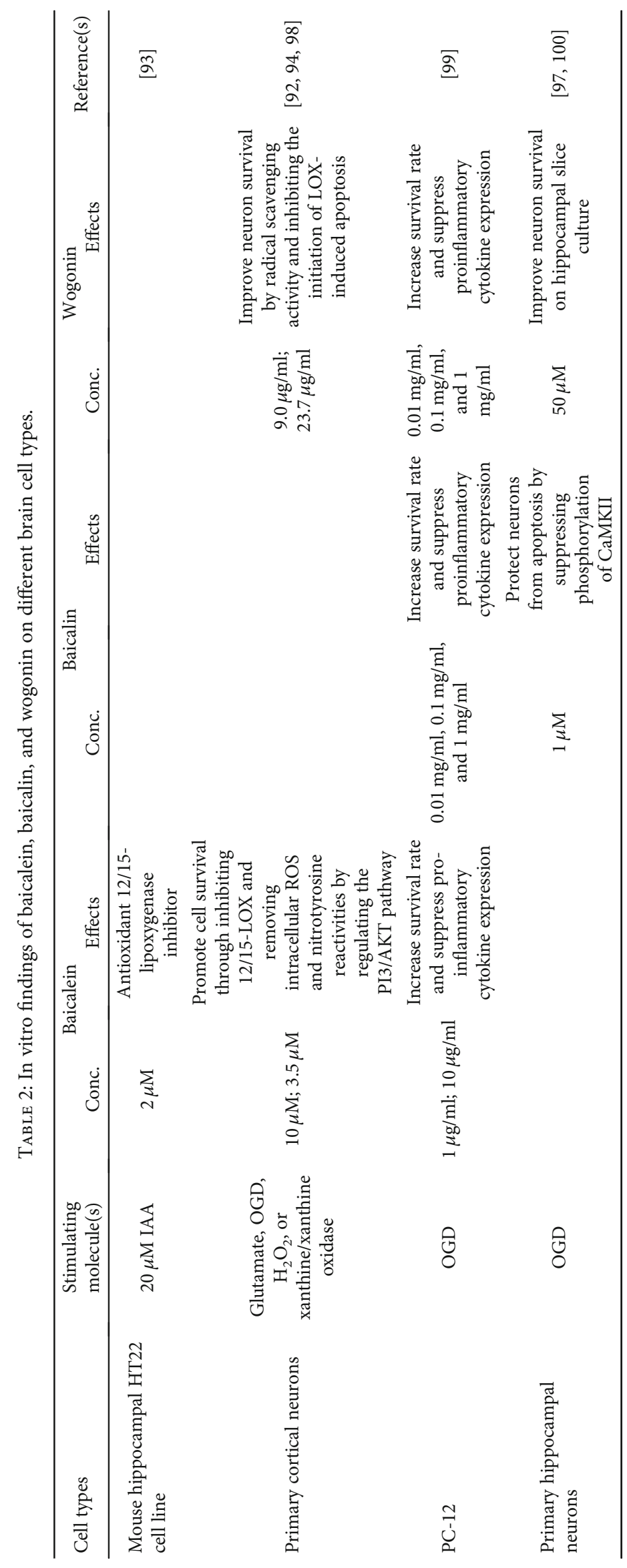




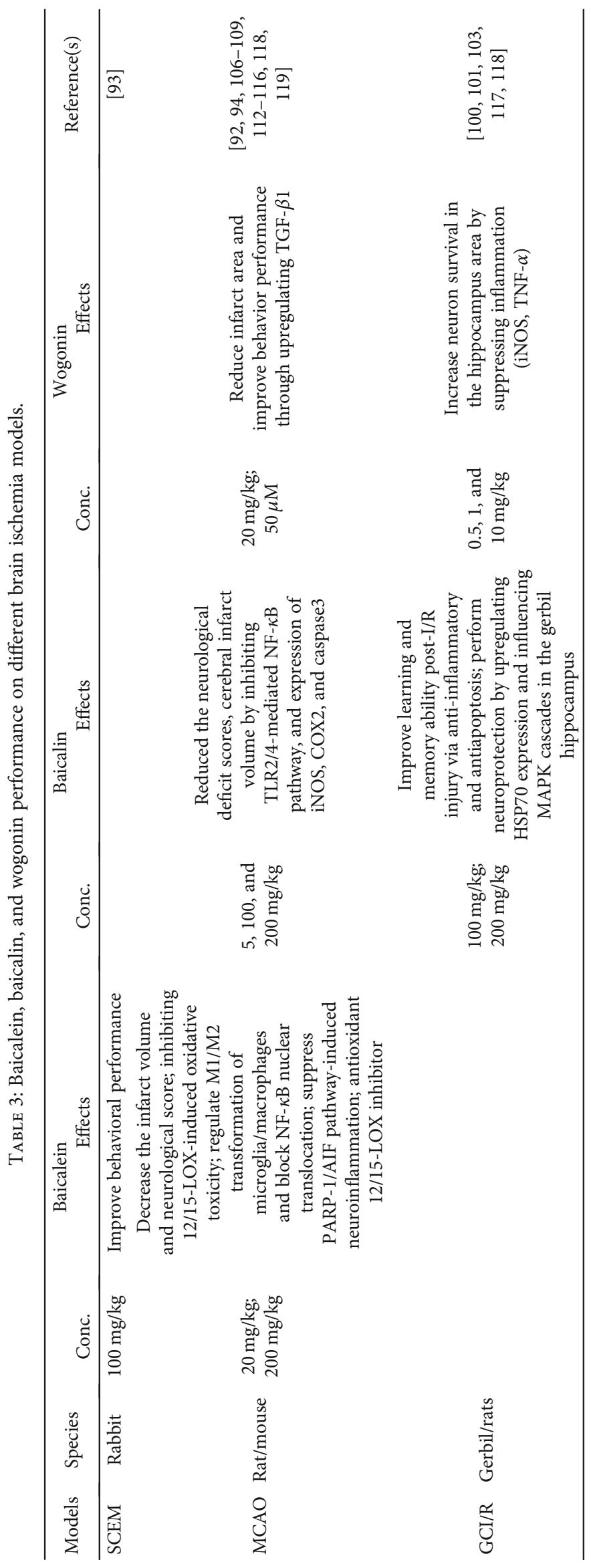




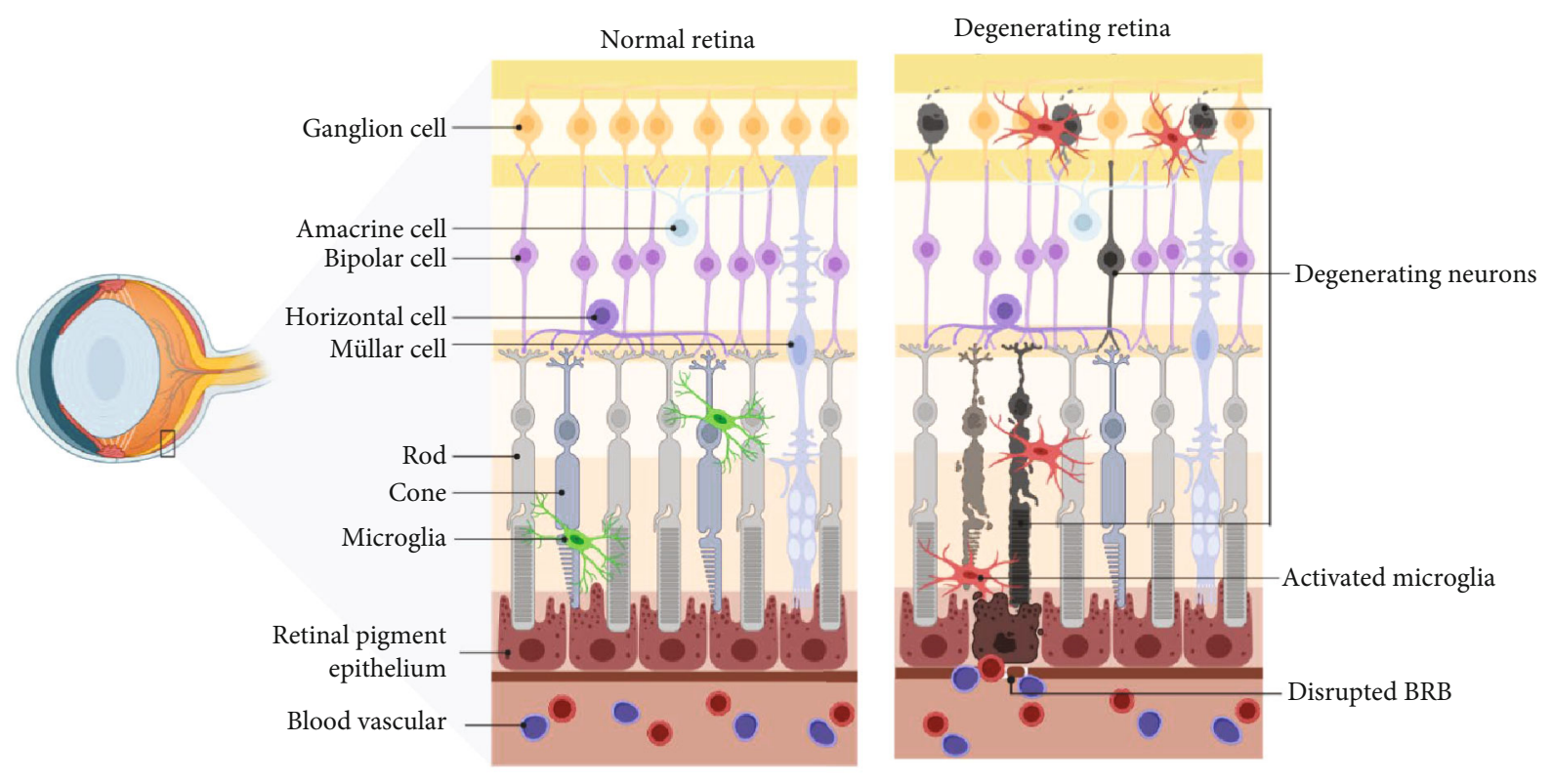

FIgURE 3: Schematic diagram showing the normal retina and degenerating retina resulting from ischemia. Created with http://BioRender.com.

reported in various animal models. In the global cerebral ischemia/reperfusion (GCI/R) rat model, Cheng et al. reported that oral administration of $100 \mathrm{mg} / \mathrm{kg}$ baicalin for $7 \mathrm{~d}$ consecutively can rescue the spatial learning and memory abilities of gerbil significantly, as assessed by the water maze test [101]. Subsequently, Wang et al. showed that baicalin, given at the same dose by intraperitoneal injection for one week immediately after GCI/R injury, can improve the learning and memory abilities in gerbil [100]. These neuroprotective effects exerted by baicalin have been found to be related to the inhibition of CaMKII-mediated downstream biochemical events [100]. CaMKII is an important protein involved in $\mathrm{Ca}^{2+} /$ glutamate-mediated excitotoxicity under the stress of ischemia $[87,102]$. These findings imply that neuroprotective effects arising from baicalin are possibly related to its antiexcitotoxicity capacity. In addition to the CaMKII pathway, Dai et al. found that neuroprotective effects of baicalin can be achieved by its mediation of heat shock protein 70 (HSP70) and mitogen-activated protein kinase (MAPKs) cascades [103]. HSP70 is a critical cytoprotective factor, which is responsible for proper protein folding [104]. And MAPKs are important pathways regulating cell survival and death, including the subgroups of phosphorylated extracellular signal-regulated kinase (pho-ERK), phosphorylated c-Jun $\mathrm{N}$-terminal kinase (pho-JNK), and phosphorylated p38 (pho-p38) [105]. Upregulation of HSP70 and mediation of MAPK subgroups by intraperitoneal administration of baicalin could effectively rescue neurons in the hippocampus after GCI/R injuries [103]. In addition, other studies have shown that baicalin can inhibit the activation of TLR signaling and the relevant downstream inflammatory pathway (i.e., nuclear factor kappa-light-chain-enhancer of activated B cells (NF$\kappa \mathrm{B})$ pathway) [106, 107]. Following middle cerebral artery occlusion (MCAO), intravenous or intraperitoneal administration of baicalin has been shown to effectively attenuate cerebral infarction by regulating inflammation, including the expression of proinflammatory cytokines TNF- $\alpha$ and interleukin- $1 \beta$ (IL- $1 \beta$ ), via TLR2/4 and NF- $\kappa$ B pathway signaling cascades $[106,107]$. Baicalin's antioxidative and antiinflammatory properties are also indicated by decreased levels of both mRNA and iNOS and COX2 protein levels $[106,108]$.

Similar neuroprotective and antineuroinflammatory effects have been reported with baicalein. For instance, baicalein was found to ameliorate the neurobehavioral deficits and infarct volume caused by small clot embolic stokes (SCEM) or MCAO [93, 94]. Modulating microglia/macrophage $\mathrm{M} 1 / \mathrm{M} 2$ polarization and suppressing the NF- $\kappa \mathrm{B}$ signaling are suggested to be responsible for the antineuroinflammation and neuroprotection [109]. As a potent antioxidant inhibitor of 12/15-LOX, baicalein was shown to effectively reduce infarct size, edema formation, and 12/15-LOXinduced neuron death in various brain ischemia animal models $[92,110-112]$. The inhibitory effect of baicalein is comparable to the protective effects observed in 12/15-LOX knockout mice ALOX15 ${ }^{-/-}$[92]. Pallast et al. found that the protective effects of baicalein are mediated by the suppression of apoptosis-inducing factor (AIF) nuclear translocation in an MCAO model [113]. Later, in addition to confirming the antiapoptotic effect through AIF regulation, $\mathrm{Li}$ et al. reported the involvement of the poly (ADP-ribose) polymerase-1 (PARP-1)/AIF pathway in baicalein-induced neuroprotection [114]. Furthermore, inflammation-related factors, such as cytosolic phospholipase A2 (cPLA2) and p38 MAPK, have been reported to be downregulated after baicalein administration in the MCAO rat model $[112,115]$. The alteration of peroxisome proliferator-activated receptor $\gamma$ $(\operatorname{PPAR} \gamma)$ and nuclear translocation induced by brain ischemia/reperfusion injury have also been reported to be returned to the balanced stage by baicalein pretreatment [116].

Wogonin is also found to exert neuroprotective effects on both GCI/R and MCAO rat models. In the GCI/R rat model, wogonin was shown to effectively attenuate neuron loss and 


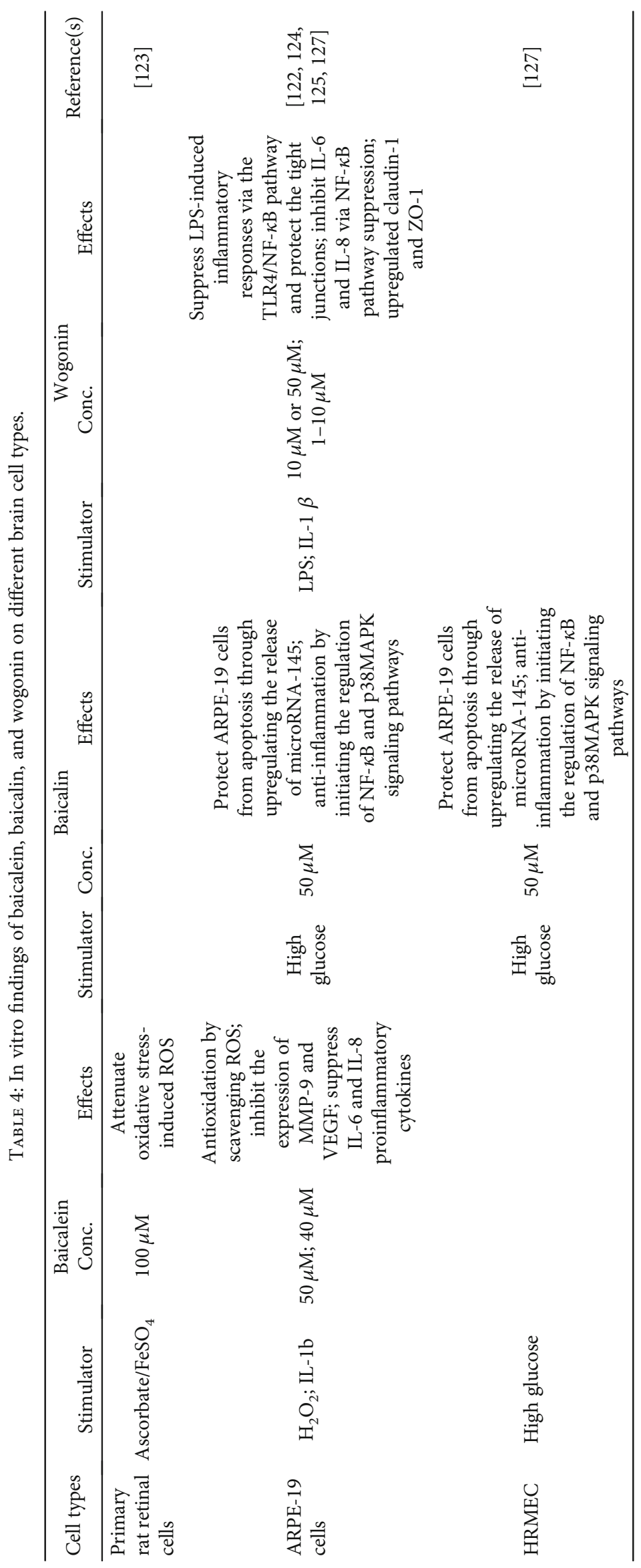


TABLE 5: In vivo findings of baicalein, baicalin, and wogonin on different animal models.

\begin{tabular}{|c|c|c|c|c|c|c|c|}
\hline \multirow{2}{*}{ Models } & \multirow{2}{*}{ Species } & \multicolumn{2}{|r|}{ Baicalein } & \multicolumn{2}{|c|}{ Baicalin } & Wogonin & \multirow{2}{*}{ References } \\
\hline & & Conc. & Effects & Conc. & Effects & Conc. Effects & \\
\hline $\mathrm{I} / \mathrm{R}$ & Rat & $0.5 \mathrm{nmol}$ & $\begin{array}{c}\text { Effectively protect retinal } \\
\text { cells and electrical functions } \\
\text { from oxidation and apoptosis; } \\
\text { upregulation of HO-1 and } \\
\text { downregulation of HIF- } 1 \alpha \text {, } \\
\text { VEGF, and MMP-1 }\end{array}$ & $12.5 \mathrm{mg} / \mathrm{kg}$ & $\begin{array}{c}\text { Protect RGCs from } \\
\text { retinal ischemia injury } \\
\text { and suppress glial cell } \\
\text { activity }\end{array}$ & & {$[123,128]$} \\
\hline DR & Rat; mice & $\begin{array}{l}150 \mathrm{mg} / \mathrm{kg} \text { in rat; } \\
75 \mathrm{mg} / \mathrm{kg} \text { in mice }\end{array}$ & $\begin{array}{l}\text { Significantly suppressed the } \\
\text { inflammatory processes of } \\
\text { retinal microglia and Muller cells; } \\
\text { enhanced vascular permeability } \\
\text { and blood-retina barrier; protect } \\
\text { BRB permeability as antioxidant } \\
\text { 12/15-LOX inhibitor, anti- } \\
\text { inflammation, and } \\
\text { antiangiogenesis }\end{array}$ & $150 \mathrm{mg} / \mathrm{ml}$ in rat & $\begin{array}{l}\text { Protect retinal cells } \\
\text { from apoptosis by } \\
\text { promoting } \\
\text { Bcl-2; perform as an } \\
\text { inhibitor of ARA and } \\
\text { delay the progression } \\
\text { of diabetic retinopathy }\end{array}$ & & {$[130,131]$} \\
\hline
\end{tabular}

histological changes in the hippocampal CA1 region [117]. The expression of inflammatory mediators (e.g., iNOS and TNF- $\alpha$ ) at the injury site was significantly suppressed by intraperitoneal administration of wogonin in rats after ischemia injury [117]. In the MCAO rat model, wogonin pre- and posttreatment was shown to both alleviate the infarct volume and behavioral deficits and promote angiogenesis in the periischemia area $[118,119]$. Upregulation of transforming growth factor beta (TGF- $\beta 1$ ) expression in the ischemic brain tissue was observed in wogonin-treated rats 2 weeks after ischemic injury [119]. TGF- $\beta 1$ has previously been reported as an important regulator of angiogenesis in hypoxic tissue [120]. This finding indicates that wogonin protects neurons by promoting microvascular formation and subsequently restoring blood supply via the TGF- $\beta 1$ pathway. The vasodilatory effect of wogonin mediated by inhibition of both intracellular $\mathrm{Ca}^{2+}$ release and extracellular $\mathrm{Ca}^{2+}$ influx could be a potential treatment paradigm for ischemia [121]. This evidence strongly supports the feasibility of developing baicalein, baicalein, and wogonin as candidates for neuroprotection following ischemic stroke.

3.2. In Retina Ischemia. Injuries caused by retinal ischemia are common in many ocular disorders, such as central/branch retinal artery/vein occlusion, DR, glaucoma, and AMD [15-19]. Similar to ischemic brain injuries, retinal ischemia triggers oxidative stress, inflammation, neovascularization, and, ultimately, the death of retinal neurons [5, 20]. Figure 3 illustrates the morphological changes to the retina and neuron death after ischemia-induced stress. In the following, the antioxidative, anti-inflammatory, antiapoptotic effects of baicalein, baicalin, and wogonin after retinal ischemia for in vitro and in vivo studies are summarized (Tables 4 and 5).

3.2.1. In Vitro Studies. The antioxidative properties of baicalein against retinal ischemia were first reported by Liu et al. [122]. Baicalein was shown to significantly increase cell viability of human retinal pigment epithelium cells (hRPEs) against $\mathrm{H}_{2} \mathrm{O}_{2}$-induced oxidative stress by scavenging ROS and suppressing the production of matrix metalloproteinase-9 (MMP-9) and vascular endothelial growth factor (VEGF) [122]. Subsequently, the antioxidative effects of baicalein on dissociated primary rat retinal cells were demonstrated to be subjected to ascorbate- and $\mathrm{FeSO}_{4}$-induced oxidative stress [123]. Baicalein pretreatment not only suppresses the expression of hypoxia-inducible factor- $1 \alpha$ (HIF- $1 \alpha)$, VEGF, and MMP-9 but also increases the level of heme oxygenase-1 (HO-1) in ascorbate- and $\mathrm{FeSO}_{4}$-stimulated retinal cells [123]. These findings indicate that baicalein has strong antioxidative capabilities in retinal cells, which are comparable to the antioxidative effects exerted by Trolox (6-hydroxy-2,5,7,8-tetramethylchroman2 -carboxylic acid) [123]. In addition, baicalein and wogonin have been found to demonstrate anti-inflammatory effects by suppressing $I L-6$ and $I L-8$ expression in IL- $1 \beta$-challenged ARPE- 19 cells, while NF- $\kappa$ B binding activity is suppressed by wogonin [124]. Similar anti-inflammatory effects have been reported by other recent studies. Chen et al. found that wogonin effectively suppressed LPS-induced activation of the TLR4/NF- $\kappa \mathrm{B}$ pathway and subsequently increased expression of inflammatory cytokines IL-1 $\beta$, IL-6, IL-8, cyclooxygenase-2 (COX-2), TNF- $\alpha$, and iNOS in ARPE-19 cells [125]. It was also reported that administration of wogonin to endoplasmic reticulum- (ER-) challenged ARPE-19 cells increased the expression of tight junction proteins claudin-1 and ZO-1 [126]. In another study which mimicked DR in vitro by treating ARPE-19 cells and human retinal microvascular endothelial cells (HRMECs) with high glucose, baicalin was shown to exert antiapoptotic effects by inhibiting the release of proinflammatory cytokines and ROS [127]. These protective effects of baicalin are likely mediated by the suppression of NF- $\kappa$ B and p38 MAPK pathways by upregulating miRNA-145 expression [127].

3.2.2. In Vivo Studies. In the rat retinal ischemia/reperfusion model, baicalein pretreatment effectively regulated the expression of apoptotic factors, including Bax and Bcl-2, subsequently reducing retinal cell apoptosis [123]. In addition, baicalein pretreatment significantly improved the inner 


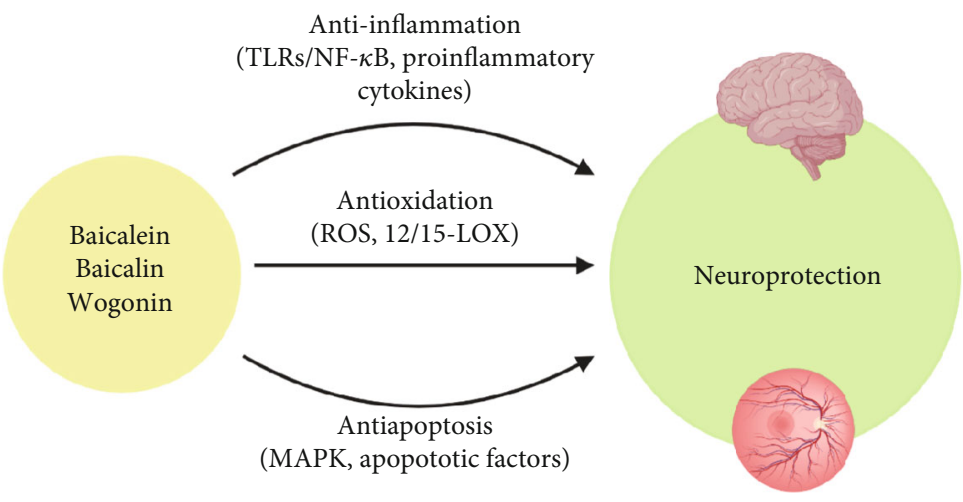

FIGURE 4: Targeted pathways initiated by baicalein, baicalin, and wogonin demonstrating the neuroprotective effects in the brain and retina. Created with http://BioRender.com.

retinal functions in electroretinogram (ERG) assessments [123]. Similar neuroprotective effects were also observed in baicalin-treated rats with ischemia/reperfusion injury. A reduced loss of Thy- $1^{+}$neuron cells and reduced expression of apoptosis markers, including caspase- 3 and 8 and poly (ADP-ribose) polymerase-1 (PARP-1), have been found in the retina after ischemic insults [128]. In addition, the downregulated expression of GFAP after baicalin treatment indicates the involvement of baicalin in regulating glial responses and neuroinflammation [128], in addition to its antioxidant and antiapoptosis properties.

The anti-inflammatory effects of baicalein and baicalin have been demonstrated in rats with DR. The excessive activation of resident retinal immune cells is widely known as a pathogenic factor of neurodegeneration in retinal disorders $[84,129]$. Yang et al. reported the occurrence of vascular abnormality and RGC loss in the DR rat retina in combination with activation of microglia and Müller cell dysfunction [130]. Oral administration of baicalein has been shown to significantly protect retinal vessels and neurons from DRinduced dysfunction and apoptosis through suppressing the activation of retinal inflammatory processes modulated by microglia and Müller cells and by reducing the release of proinflammatory cytokines, including IL-18, TNF- $\alpha$, and IL- $1 \beta$ [130]. The protective effects of baicalin are believed to function through inhibiting the expression of apoptosis regulators, including $\mathrm{Bax}$ and $\mathrm{Bcl}-2$, on the RGC layer [131]. Intraperitoneal application of baicalin was found to inhibit the otherwise-elevated aldose reductase activity (ARA) in diabetes. This suggests that baicalin acts as an aldose reductase inhibitor, potentially retarding the progression of apoptosis induced by diabetes [131].

In addition to oxidative stress and inflammation, vascular hyperpermeability of retinal blood vessels has been suggested to be a pathogenic factor in retinal ischemia, DR, and AMD. Othman et al. reported that 12/15-LOX activation leads to vascular hyperpermeability in DR by inhibiting protein tyrosine phosphatase and activating VEGF-R2 signaling pathways [132]. As a potent inhibitor of 12/15-LOX, baicalein significantly reduces the lipid metabolites of 12 - and 15hydroxyeicosatetreanoic acids (HETE) expressed during 12/15-LOX activation in DR [132]. The HETE-induced upregulation of NOX2 and ROS is also reported to be down- regulated by baicalein treatment in Ins2 $2^{\text {Akita }}$ diabetic mouse retina. Likewise, baicalein has been shown to protect against HETE-induced vascular hyperpermeability by acting as a VEGF-R2 inhibitor, restoring phosphoserine phosphatase-1 (pSHP1) levels in DR [132]. The inflammatory cytokine IL6 and intracellular adhesion molecules (ICAMs) ICAM-1 and vascular cell adhesion molecule-1 (VCAM-1) were remarkably inhibited in the diabetic retina [132]. Taken together, baicalein functions as a $12 / 15$-LOX inhibitor, mediating its effects primarily on vascular and retinal barriers.

\section{Pathways Targeted by Baicalein, Baicalin, and Wogonin during Neuroprotective Processes}

As described above, the neuroprotective effects triggered by baicalein, baicalin, and wogonin are possibly related to their anti-inflammatory, antioxidative, and antiapoptotic capabilities (Figure 4).

Firstly, the antioxidative effects of baicalein, baicalin, and wogonin are recognized by their ROS scavenging properties. Under normal circumstance, ROS play a pivotal role in many biological processes, such as redox balance in cells. However, a dramatic increase of ROS production may disturb this homeostatic balance under oxidative stress conditions (e.g., ischemia), eventually leading to cell death. Typically, scavenging excessive ROS is a neuroprotective target in neurodegenerative disorders [133]. Baicalein and baicalin serve as potent $12 / 15$-LOX inhibitors with high antioxidative efficiencies. 12/15-LOX is found to be upregulated after stroke, resulting in neuronal death and leakage of $\mathrm{BBB}$ and $\mathrm{BRB}$ [134]. Inhibition of 12/15-LOX was also shown to reduce infarct volume and edema in the stroke area, suggesting its potential role as an effective and viable therapeutic option for ischemia $[92,135,136]$. Furthermore, the protection of $\mathrm{BBB}$ and $\mathrm{BRB}$ help preserves the microenvironment at the injured site from disruption by systemic immunity and secondary inflammatory responses caused by degeneration.

Secondly, the anti-inflammatory effect initiated by these flavonoids after ischemia is revealed by the suppression of proinflammatory cytokine release. Excessive activation of microglia has been emergingly reported as a pathogenesis for the development of neurodegenerative diseases [137]. In addition, baicalein and baicalin exhibit the capability in 
regulating microglia homeostasis after ischemic stress [109]. These properties have been demonstrated to be mediated through interactions with TLR/NF- $\kappa \mathrm{B}$ and PARP-1/AIF pathways [99, 106, 107, 124, 125, 127].

Lastly, the antiapoptotic capacity of baicalein, baicalin, and wogonin has been reported in ischemia-injured brain and retina. Through modulating the MAPK pathway and the production of apoptotic factors, these flavonoids effectively rescue neurons in the brain and retina $[100,112,113$, $115,123,127,128,131]$. In addition, the blockage of ion channel dysfunction after ischemic stress remarkably ameliorates the excitotoxicity caused by ion overload and subsequently decreases neuronal death $[86,121]$. Taken together, all the evidence strongly supports the feasibility of developing these flavonoids as natural neuroprotectants.

\section{Conclusion and Future Directions}

Ischemia often results in physical disabilities, including paralysis and blindness. This is particularly common in the aging population. Currently, effective clinical intervention for ischemia-induced damage is limited. Ample evidence has demonstrated the potent neuroprotective properties of baicalein, baicalin, and wogonin in both in vitro and in vivo models of ischemia. Flavonoids exert their antiinflammatory, antioxidative, and antiapoptotic effects on CNS postischemia insults by initiating various potential signaling pathways. Flavonoids are herb extracts, suggesting their potential to be developed as natural neuroprotective agents.

Additional studies are required to elucidate and characterize the pharmacological properties and bioactivities of these flavonoids in combating neuropathies, in order to facilitate the future development of neuroprotectants that are safe and effective. As an extension of the brain, the retina may serve as an easily accessible model and potential therapeutic target site for various neuropathies and other neurodegenerative diseases. Additionally, pharmacodynamic and pharmacokinetic studies, including time- and dose-dependent responses, cytotoxicity, and drug metabolism after systemic and topical administration of these three flavonoids, need to be established. One recent study has reported that the metabolic abilities of flavonoids in the liver and intestines are markedly different among different species, including mice, rats, dogs, monkeys, and humans [138]. In addition, potential targets or receptors through which baicalein, baicalin, and wogonin act on may need to be further characterized and studied. Studies on the combined effects of baicalein, baicalin, and wogonin are limited, raising the possibility that combined flavonoids can achieve longer and stronger protective effects. Lastly, due to the limited water solubility and liposolubility of baicalein, baicalin, and wogonin, formulations and optimizations of these flavonoids, possibly including nanoparticles or other newly developed carriers, may be needed to achieve higher bioactivity and clinical efficacy. It is envisaged that these natural flavonoids can eventually offer new therapeutic therapies for patients with ischemia-induced neural disorders.

\section{Data Availability}

All data mentioned in this review article are published findings. They have been properly cited in the article.

\section{Conflicts of Interest}

D.F.C. is a consultant for Boston Pharma and Pri-Med. Other authors have no conflicts of interest.

\section{Acknowledgments}

This work was supported by grants from the National Institutes of Health (NIH)/National Eye Institute (NEI) (Grants EY025913 and EY025259 to D.F.C.), Bright Focus Foundation grant and The Glaucoma Foundation grant to K.C., the Core Grant for Vision Research from NIH/NEI to the Schepens Eye Research Institute (P30EY03790), Health Medical Research Fund (16172571 to C.W.D), PolyU Postgraduate Studentship (L.P.), Henry G. Leong Endowed Professorship in Elderly Vision Health and Dean Reserve (8-8475 and 1ZVN2 to C.H.T), and PolyU internal grants (UAGF and UAHG to C.W.D).

\section{References}

[1] H. K. Eltzschig and T. Eckle, "Ischemia and reperfusion-from mechanism to translation," Nature Medicine, vol. 17, no. 11, pp. 1391-1401, 2011.

[2] H. Amani, E. Mostafavi, M. R. Alebouyeh et al., "Would colloidal gold nanocarriers present an effective diagnosis or treatment for ischemic stroke?," International Journal of Nanomedicine, vol. 14, pp. 8013-8031, 2019.

[3] H. Amani, R. Habibey, F. Shokri et al., "Selenium nanoparticles for targeted stroke therapy through modulation of inflammatory and metabolic signaling," Scientific Reports, vol. 9, no. 1, article 6044, 2019.

[4] R. Capildeo, S. Haberman, and F. C. Rose, "The definition and classification of stroke: a new approach," The Quarterly Journal of Medicine, vol. 47, no. 2, pp. 177-196, 1978.

[5] P. Deb, S. Sharma, and K. M. Hassan, "Pathophysiologic mechanisms of acute ischemic stroke: an overview with emphasis on therapeutic significance beyond thrombolysis," Pathophysiology, vol. 17, no. 3, pp. 197-218, 2010.

[6] Writing Group Members, T. Thom, N. Haase et al., "Heart disease and stroke statistics-2006 Update," Circulation, vol. 113, no. 6, pp. e85-e151, 2006.

[7] B. Boling and K. Keinath, "Acute ischemic stroke," AACN Advanced Critical Care, vol. 29, no. 2, pp. 152-162, 2018.

[8] Q. Yang, X. Tong, L. Schieb et al., "Vital signs: recent trends in stroke death rates - United States, 2000-2015," MMWR Morbidity and Mortality Weekly Report, vol. 66, no. 35, pp. 933-939, 2017.

[9] J. S. Kim, "tPA helpers in the treatment of acute ischemic stroke: are they ready for clinical use?," Journal of Stroke, vol. 21, no. 2, pp. 160-174, 2019.

[10] V. K. Sharma, H. L. Teoh, L. Y. H. Wong, J. Su, B. K. C. Ong, and B. P. L. Chan, "Recanalization therapies in acute ischemic stroke: pharmacological agents, devices, and combinations," Stroke Research and Treatment, vol. 2010, Article ID 672064, 8 pages, 2010. 
[11] J. K. Mai and G. Paxinos, The Human Nervous System, Academic Press, Cambridge, MA, USA, 2011.

[12] J. J. Pang, F. Gao, and S. M. Wu, "Light-evoked excitatory and inhibitory synaptic inputs to ON and OFF $\alpha$ ganglion cells in the mouse retina," The Journal of Neuroscience, vol. 23, no. 14, pp. 6063-6073, 2003.

[13] M. Tessier-Lavigne, "Visual processing by the retina," in Principles of Neural Science, pp. 507-522, Elsevier, 2000.

[14] S. Prasad and S. L. Galetta, "Chapter 1 - Anatomy and physiology of the afferent visual system," in Handbook of Clinical Neurology, C. Kennard and R. J. Leigh, Eds., pp. 3-19, Elsevier, 2011.

[15] Y. Q. Chen, W. H. T. Pan, J. H. Liu et al., "The effects and underlying mechanisms ofS-Allyll-Cysteine treatment of the retina after ischemia/reperfusion," Journal of Ocular Pharmacology and Therapeutics, vol. 28, no. 2, pp. 110-117, 2012.

[16] P. H. Peng, H. M. Chao, S. H. Juan, C. F. Chen, J. H. Liu, and M. L. Ko, "Pharmacological preconditioning by low dose cobalt protoporphyrin induces heme oxygenase- 1 overexpression and alleviates retinal ischemia-reperfusion injury in rats," Current Eye Research, vol. 36, no. 3, pp. 238-246, 2011.

[17] M. M. Wessel, N. Nair, G. D. Aaker, J. R. Ehrlich, D. J. D'Amico, and S. Kiss, "Peripheral retinal ischaemia, as evaluated by ultra-widefield fluorescein angiography, is associated with diabetic macular oedema," The British Journal of Ophthalmology, vol. 96, no. 5, pp. 694-698, 2012.

[18] N. N. Osborne, M. Ugarte, M. Chao et al., "Neuroprotection in relation to retinal ischemia and relevance to glaucoma," Survey of Ophthalmology, vol. 43, pp. S102-S128, 1999.

[19] N. N. Osborne, R. J. Casson, J. P. M. Wood, G. Chidlow, M. Graham, and J. Melena, "Retinal ischemia: mechanisms of damage and potential therapeutic strategies," Progress in Retinal and Eye Research, vol. 23, no. 1, pp. 91-147, 2004.

[20] T. T. Lam, A. S. Abler, and M. Tso, "Apoptosis and caspases after ischemia-reperfusion injury in rat retina," Investigative Ophthalmology \& Visual Science, vol. 40, no. 5, pp. 967975, 1999.

[21] I. J. MacCormick, G. Czanner, and B. Faragher, "Developing retinal biomarkers of neurological disease: an analytical perspective," Biomarkers in Medicine, vol. 9, no. 7, pp. 691701, 2015.

[22] A. London, I. Benhar, and M. Schwartz, "The retina as a window to the brain-from eye research to CNS disorders," Nature Reviews Neurology, vol. 9, no. 1, pp. 44-53, 2013.

[23] M. H. Madeira, A. F. Ambrósio, and A. R. Santiago, "Gliamediated retinal neuroinflammation as a biomarker in Alzheimer's disease," Ophthalmic Research, vol. 54, no. 4, pp. 204-211, 2015.

[24] J. A. Fernández-Albarral, E. Salobrar-García, R. MartínezPáramo et al., "Cambios de las celulas gliales de la retina en la enfermedad de Alzheimer - Revision bibliografica," Journal of Optometry, vol. 12, no. 3, pp. 198-207, 2019.

[25] J. Y. Niederkorn, "See no evil, hear no evil, do no evil: the lessons of immune privilege," Nature Immunology, vol. 7, no. 4, pp. 354-359, 2006.

[26] C. Arcuri, C. Mecca, R. Bianchi, I. Giambanco, and R. Donato, "The pathophysiological role of microglia in dynamic surveillance, phagocytosis and structural remodeling of the developing CNS," Frontiers in Molecular Neuroscience, vol. 10, no. 191, 2017.
[27] T. H. Khanh Vu, H. Chen, L. Pan et al., "CD4 ${ }^{+}$T-Cell Responses Mediate Progressive Neurodegeneration in Experimental Ischemic Retinopathy," The American Journal of Pathology, vol. 190, no. 8, pp. 1723-1734, 2020.

[28] A. Murshid, J. Gong, and S. K. Calderwood, "The role of heat shock proteins in antigen cross presentation," Frontiers in Immunology, vol. 3, p. 63, 2012.

[29] S. Jiang, M. Kametani, and D. F. Chen, "Adaptive immunity: new aspects of pathogenesis underlying neurodegeneration in glaucoma and optic neuropathy," Frontiers in Immunology, vol. 11, p. 65, 2020.

[30] T. Peng, Y. Jiang, M. Farhan, P. Lazarovici, L. Chen, and W. Zheng, "Anti-inflammatory effects of traditional chinese medicines on preclinical in vivo models of brain ischemiareperfusion-injury: prospects for neuroprotective drug discovery and therapy," Frontiers in Pharmacology, vol. 10, no. 204, 2019.

[31] J. H. Park, O. . Park, J. H. Cho et al., “Anti-inflammatory effect of tanshinone I in neuroprotection against cerebral ischemia-reperfusion injury in the gerbil hippocampus," Neurochemical Research, vol. 39, no. 7, pp. 1300-1312, 2014.

[32] K. Dong, W. Xu, J. Yang, H. Qiao, and L. Wu, "Neuroprotective effects of Tanshinone IIA on permanent focal cerebral ischemia in mice," Phytotherapy Research, vol. 23, no. 5, pp. 608-613, 2009.

[33] C. Ling, J. Liang, C. Zhang et al., "Synergistic effects of salvianolic acid B and puerarin on cerebral ischemia reperfusion injury," Molecules, vol. 23, no. 3, p. 564, 2018.

[34] X. Fang, Y. Li, J. Qiao, Y. Guo, and M. Miao, "Neuroprotective effect of total flavonoids from Ilex pubescens against focal cerebral ischemia/reperfusion injury in rats," Molecular Medicine Reports, vol. 16, no. 5, pp. 7439-7449, 2017.

[35] Y. Yuan, M. Fang, C. Y. Wu, and E. A. Ling, "Scutellarin as a potential therapeutic agent for microglia-mediated neuroinflammation in cerebral ischemia," Neuromolecular Medicine, vol. 18, no. 3, pp. 264-273, 2016.

[36] C. Y. Wu, M. Fang, A. Karthikeyan, Y. Yuan, and E. A. Ling, "Scutellarin attenuates microglia-mediated neuroinflammation and promotes astrogliosis in cerebral ischemia-a therapeutic consideration," Current Medicinal Chemistry, vol. 24, no. 7, pp. 718-727, 2017.

[37] A. Wąsik and L. Antkiewicz-Michaluk, "The mechanism of neuroprotective action of natural compounds," Pharmacological Reports, vol. 69, no. 5, pp. 851-860, 2017.

[38] M. Daglia, A. Lorenzo, S. Nabavi, Z. Talas, and S. Nabavi, "Polyphenols: well beyond the antioxidant capacity: gallic acid and related compounds as neuroprotective agents: you are what you eat!," Current Pharmaceutical Biotechnology, vol. 15, no. 4, pp. 362-372, 2014.

[39] I. E. Orhan, M. Daglia, S. F. Nabavi, M. R. Loizzo, E. SobarzoSanchez, and S. M. Nabavi, "Flavonoids and dementia: an update," Current Medicinal Chemistry, vol. 22, no. 8, pp. 1004-1015, 2015.

[40] A. Y. Chen and Y. C. Chen, "A review of the dietary flavonoid, kaempferol on human health and cancer chemoprevention," Food Chemistry, vol. 138, no. 4, pp. 2099-2107, 2013.

[41] N. S. Alrawaiq and A. Abdullah, "A review of flavonoid quercetin: metabolism, bioactivity and antioxidant properties," International Journal of PharmTech Research, vol. 6, no. 3, pp. 933-941, 2014. 
[42] H. Hosseinzadeh and M. Nassiri-Asl, "Review of the protective effects of rutin on the metabolic function as an important dietary flavonoid," Journal of Endocrinological Investigation, vol. 37, no. 9, pp. 783-788, 2014.

[43] A. Liu, W. Wang, H. Fang et al., "Baicalein protects against polymicrobial sepsis-induced liver injury via inhibition of inflammation and apoptosis in mice," European Journal of Pharmacology, vol. 748, pp. 45-53, 2015.

[44] X. Ma, W. Yan, Z. Dai et al., "Baicalein suppresses metastasis of breast cancer cells by inhibiting EMT via downregulation of SATB1 and Wnt/ $\beta$-catenin pathway," Drug Design, Development and Therapy, vol. 10, article 1419, 2016.

[45] Z. Huang, X. Pan, J. Zhou, W. T. Leung, C. Li, and L. Wang, "Chinese herbal medicine for acute upper respiratory tract infections and reproductive safety: a systematic review," Bioscience Trends, vol. 13, no. 2, pp. 117-129, 2019.

[46] M. J. Bae, H. S. Shin, H. J. See, S. Y. Jung, D. A. Kwon, and D. H. Shon, "Baicalein induces $\mathrm{CD} 4^{+} \mathrm{Foxp}^{+} \mathrm{T}$ cells and enhances intestinal barrier function in a mouse model of food allergy," Scientific Reports, vol. 6, no. 1, pp. 1-11, 2016.

[47] Y. Amakura, A. Yoshimura, M. Yoshimura, and T. Yoshida, "Isolation and characterization of phenolic antioxidants from Plantago herb," Molecules, vol. 17, no. 5, pp. 5459-5466, 2012.

[48] Q. Cong, M. Shang, Q. Dong, W. Liao, F. Xiao, and K. Ding, "Structure and activities of a novel heteroxylan from Cassia obtusifolia seeds and its sulfated derivative," Carbohydrate Research, vol. 393, pp. 43-50, 2014.

[49] J. Ming, L. Zhuoneng, and Z. Guangxun, "Protective role of flavonoid baicalin from Scutellaria baicalensis in periodontal disease pathogenesis: A literature review," Complementary Therapies in Medicine, vol. 38, pp. 11-18, 2018.

[50] W. Y. Gong, Z. X. Zhao, B. J. Liu, L. W. Lu, and J. C. Dong, "Exploring the chemopreventive properties and perspectives of baicalin and its aglycone baicalein in solid tumors," European Journal of Medicinal Chemistry, vol. 126, pp. 844-852, 2017.

[51] J. R. Xiao, C. W. Do, and C. H. To, "Potential therapeutic effects of baicalein, baicalin, and wogonin in ocular disorders," Journal of Ocular Pharmacology and Therapeutics, vol. 30, no. 8, pp. 605-614, 2014.

[52] B. Dinda, S. Dinda, S. DasSharma, R. Banik, A. Chakraborty, and M. Dinda, "Therapeutic potentials of baicalin and its aglycone, baicalein against inflammatory disorders," European Journal of Medicinal Chemistry, vol. 131, pp. 68-80, 2017.

[53] J. Ru, P. Li, J. Wang et al., "TCMSP: a database of systems pharmacology for drug discovery from herbal medicines," Journal of Cheminformatics, vol. 6, no. 1, pp. 1-6, 2014.

[54] P. Matsson and J. Kihlberg, "How big is too big for cell permeability?," Journal of Medicinal Chemistry, vol. 60, no. 5, pp. 1662-1664, 2017.

[55] P. Ertl, B. Rohde, and P. Selzer, "Fast calculation of molecular polar surface area as a sum of fragment-based contributions and its application to the prediction of drug transport properties," Journal of Medicinal Chemistry, vol. 43, no. 20, pp. 3714-3717, 2000.

[56] M. H. N. Tattersall, J. E. Sodergren, S. K. Sengupta, D. H. Trites, E. J. Modest, and E. Frei III, "Pharmacokinetics of actinomycin 0 in patients with malignant melanoma," Clinical Pharmacology and Therapeutics, vol. 17, no. 6, pp. 701-708, 1975.
[57] W. Tao, X. Xu, X. Wang et al., "Network pharmacologybased prediction of the active ingredients and potential targets of Chinese herbal Radix Curcumae formula for application to cardiovascular disease," Journal of Ethnopharmacology, vol. 145, no. 1, pp. 1-10, 2013.

[58] V. N. Viswanadhan, A. K. Ghose, G. R. Revankar, and R. K. Robins, "Atomic physicochemical parameters for three dimensional structure directed quantitative structureactivity relationships. 4. Additional parameters for hydrophobic and dispersive interactions and their application for an automated superposition of certain naturally occurring nucleoside antibiotics," Journal of Chemical Information and Modeling, vol. 29, no. 3, pp. 163-172, 1989.

[59] J. Luo, H. Kong, M. Zhang et al., "Novel carbon dots-derived fromRadix PuerariaeCarbonisata significantly improve the solubility and bioavailability of baicalin," Journal of Biomedical Nanotechnology, vol. 15, no. 1, pp. 151-161, 2019.

[60] G. Zahra, B. Khadijeh, K. Mortaza, and S. Ali, "Potential therapeutic effects and bioavailability of wogonin, the flavone of Baikal skullcap," Journal of Nutritional Medicine and Diet Care, vol. 5, no. 2, p. 39, 2019.

[61] Y. Gao, S. A. Snyder, J. N. Smith, and Y. C. Chen, "Anticancer properties of baicalein: a review," Medicinal Chemistry Research, vol. 25, no. 8, pp. 1515-1523, 2016.

[62] R. Muto, T. Motozuka, M. Nakano, Y. Tatsumi, F. Sakamoto, and N. Kosaka, "The chemical structure of new substance as the metabolite of baicalin and time profiles for the plasma concentration after oral administration of sho-saiko-to in human," Yakugaku Zasshi, vol. 118, no. 3, pp. 79-87, 1998.

[63] M. Y. Lai, Y. C. Hou, S. L. Hsiu, C. C. Chen, and P. D. L. Chao, "Relative flavone bioavailability of Scutellariae Radix between traditional decoction and commercial powder preparation in humans," Journal of Food and Drug Analysis, vol. 10, no. 2, 2002.

[64] S. Tian, G. He, J. Song et al., "Pharmacokinetic study of baicalein after oral administration in monkeys," Fitoterapia, vol. 83, no. 3, pp. 532-540, 2012.

[65] T. Huang, Y. Liu, and C. Zhang, "Pharmacokinetics and bioavailability enhancement of baicalin: a review," European Journal of Drug Metabolism and Pharmacokinetics, vol. 44, no. 2, pp. 159-168, 2019.

[66] Y. Tang, H. Zhu, Y. Zhang, and C. Huang, "Determination of human plasma protein binding of baicalin by ultrafiltration and high-performance liquid chromatography," Biomedical Chromatography, vol. 20, no. 10, pp. 1116-1119, 2006.

[67] H. Pang, W. Xue, A. Shi et al., "Multiple-ascending-dose pharmacokinetics and safety evaluation of baicalein chewable tablets in healthy Chinese volunteers," Clinical Drug Investigation, vol. 36, no. 9, pp. 713-724, 2016.

[68] M. Li, A. Shi, H. Pang et al., "Safety, tolerability, and pharmacokinetics of a single ascending dose of baicalein chewable tablets in healthy subjects," Journal of Ethnopharmacology, vol. 156, pp. 210-215, 2014.

[69] W. W. Chen, X. Zhang, and W. J. Huang, "Role of neuroinflammation in neurodegenerative diseases (Review)," Molecular Medicine Reports, vol. 13, no. 4, pp. 3391-3396, 2016.

[70] S. DiMauro and E. A. Schon, "Mitochondrial disorders in the nervous system," Annual Review of Neuroscience, vol. 31, no. 1, pp. 91-123, 2008. 
[71] R. Lardenoije, A. Iatrou, G. Kenis et al., "The epigenetics of aging and neurodegeneration," Progress in Neurobiology, vol. 131, pp. 21-64, 2015.

[72] A. Ciechanover and Y. T. Kwon, "Degradation of misfolded proteins in neurodegenerative diseases: therapeutic targets and strategies," Experimental \& Molecular Medicine, vol. 47, no. 3, article e147, 2015.

[73] Z. Liu, T. Zhou, A. C. Ziegler, P. Dimitrion, and L. Zuo, "Oxidative stress in neurodegenerative diseases: from molecular mechanisms to clinical applications," Oxidative Medicine and Cellular Longevity, vol. 2017, Article ID 2525967, 11 pages, 2017.

[74] M. Vila and S. Przedborski, "Targeting programmed cell death in neurodegenerative diseases," Nature Reviews Neuroscience, vol. 4, no. 5, pp. 365-375, 2003.

[75] G. A. Donnan, M. Fisher, M. Macleod, and S. M. Davis, “Stroke," Lancet, vol. 371, no. 9624, pp. 1612-1623, 2008.

[76] H. Z. Xu and Y. Z. Le, "Significance of outer blood-retina barrier breakdown in diabetes and ischemia," Investigative Ophthalmology \& Visual Science, vol. 52, no. 5, pp. 2160-2164, 2011.

[77] U. Dirnagl, C. Iadecola, and M. A. Moskowitz, "Pathobiology of ischaemic stroke: an integrated view," Trends in Neurosciences, vol. 22, no. 9, pp. 391-397, 1999.

[78] B. P. Gaire, "Herbal medicine in ischemic stroke: challenges and prospective," Chinese Journal of Integrative Medicine, vol. 24, no. 4, pp. 243-246, 2018.

[79] H. Chen, H. Yoshioka, G. S. Kim et al., "Oxidative stress in ischemic brain damage: mechanisms of cell death and potential molecular targets for neuroprotection," Antioxidants \& Redox Signaling, vol. 14, no. 8, pp. 1505-1517, 2011.

[80] H. M. Honda, P. Korge, and J. N. Weiss, "Mitochondria and ischemia/reperfusion injury," Annals of the New York Academy of Sciences, vol. 1047, no. 1, pp. 248-258, 2005.

[81] T. M. Woodruff, J. Thundyil, S.-C. Tang, C. G. Sobey, S. M. Taylor, and T. V. Arumugam, "Pathophysiology, treatment, and animal and cellular models of human ischemic stroke," Molecular Neurodegeneration, vol. 6, no. 1, p. 11, 2011.

[82] L. Guzman-Martinez, R. B. Maccioni, V. Andrade, L. P. Navarrete, M. G. Pastor, and N. Ramos-Escobar, "Neuroinflammation as a common feature of neurodegenerative disorders," Frontiers in Pharmacology, vol. 10, article 1008, 2019.

[83] R. L. Jayaraj, S. Azimullah, R. Beiram, F. Y. Jalal, and G. A. Rosenberg, "Neuroinflammation: friend and foe for ischemic stroke," Journal of Neuroinflammation, vol. 16, no. 1, p. 142, 2019.

[84] H. S. Kwon and S. H. Koh, "Neuroinflammation in neurodegenerative disorders: the roles of microglia and astrocytes," Translational Neurodegeneration, vol. 9, no. 1, p. 42, 2020.

[85] H. Zhang, X. Xiong, L. Gu, W. Xie, and H. Zhao, "CD4 T cell deficiency attenuates ischemic stroke, inhibits oxidative stress, and enhances Akt/mTOR survival signaling pathways in mice," Chinese Neurosurgical Journal, vol. 4, no. 1, p. 32, 2018.

[86] A. Khanna, K. T. Kahle, B. P. Walcott, V. Gerzanich, and J. M. Simard, "Disruption of ion homeostasis in the neurogliovascular unit underlies the pathogenesis of ischemic cerebral edema," Translational Stroke Research, vol. 5, no. 1, pp. 316, 2014.

[87] S. J. Coultrap, R. S. Vest, N. M. Ashpole, A. Hudmon, and K. U. Bayer, "CaMKII in cerebral ischemia," Acta Pharmacologica Sinica, vol. 32, no. 7, pp. 861-872, 2011.
[88] A. W. Siu, M. K. Lau, J. S. Cheng et al., "Glutamate-induced retinal lipid and protein damage: the protective effects of catechin," Neuroscience Letters, vol. 432, no. 3, pp. 193-197, 2008.

[89] T. Tao, M. Liu, M. Chen et al., "Natural medicine in neuroprotection for ischemic stroke: challenges and prospective," Pharmacology \& Therapeutics, vol. 216, article 107695, 2020.

[90] D. Radak, N. Katsiki, I. Resanovic et al., "Apoptosis and acute brain ischemia in ischemic stroke," Current Vascular Pharmacology, vol. 15, no. 2, pp. 115-122, 2017.

[91] M. Yousufuddin and N. Young, "Aging and ischemic stroke," Aging, vol. 11, no. 9, pp. 2542-2544, 2019.

[92] K. van Leyen, H. Y. Kim, S. R. Lee, G. Jin, K. Arai, and E. H. Lo, "Baicalein and 12/15-lipoxygenase in the ischemic brain," Stroke, vol. 37, no. 12, pp. 3014-3018, 2006.

[93] P. A. Lapchak, P. Maher, D. Schubert, and J. A. Zivin, "Baicalein, an antioxidant 12/15-lipoxygenase inhibitor improves clinical rating scores following multiple infarct embolic strokes," Neuroscience, vol. 150, no. 3, pp. 585-591, 2007.

[94] C. Liu, J. Wu, K. Xu et al., "Neuroprotection by baicalein in ischemic brain injury involves PTEN/AKT pathway," Journal of Neurochemistry, vol. 112, no. 6, pp. 1500-1512, 2010.

[95] H. Kuhn, S. Banthiya, and K. van Leyen, "Mammalian lipoxygenases and their biological relevance," Biochimica et Biophysica Acta (BBA) - Molecular and Cell Biology of Lipids, vol. 1851, no. 4, pp. 308-330, 2015.

[96] N. K. Singh and G. N. Rao, "Emerging role of 12/15-lipoxygenase (ALOX15) in human pathologies," Progress in Lipid Research, vol. 73, pp. 28-45, 2019.

[97] D. Son, P. Lee, J. Lee, H. Kim, and S. Y. Kim, "Neuroprotective effect of wogonin in hippocampal slice culture exposed to oxygen and glucose deprivation," European Journal of Pharmacology, vol. 493, no. 1-3, pp. 99-102, 2004.

[98] J. Cho and H. K. Lee, "Wogonin inhibits excitotoxic and oxidative neuronal damage in primary cultured rat cortical cells," European Journal of Pharmacology, vol. 485, no. 1-3, pp. 105-110, 2004.

[99] H. Y. Li, J. Hu, S. Zhao et al., "Comparative Study of the Effect of Baicalin and Its Natural Analogs on Neurons with Oxygen and Glucose Deprivation Involving Innate Immune Reaction of TLR2/TNF $\alpha$," Journal of Biomedicine and Biotechnology, vol. 2012, Article ID 267890, 9 pages, 2012.

[100] P. Wang, Y. Cao, J. Yu et al., "Baicalin alleviates ischemiainduced memory impairment by inhibiting the phosphorylation of CaMKII in hippocampus," Brain Research, vol. 1642, pp. 95-103, 2016.

[101] O. Cheng, Z. Li, Y. Han, Q. Jiang, Y. Yan, and K. Cheng, "Baicalin improved the spatial learning ability of global ischemia/reperfusion rats by reducing hippocampal apoptosis," Brain Research, vol. 1470, pp. 111-118, 2012.

[102] H. T. Lu, R. Q. Feng, J. K. Tang, J. J. Zhou, F. Gao, and J. Ren, "CaMKII/calpain interaction mediates ischemia/reperfusion injury in isolated rat hearts," Cell Death \& Disease, vol. 11, no. 5 , p. $388,2020$.

[103] J. Dai, L. Chen, Y. M. Qiu et al., “Activations of GABAergic signaling, HSP70 and MAPK cascades are involved in baicalin's neuroprotection against gerbil global ischemia/reperfusion injury," Brain Research Bulletin, vol. 90, pp. 1-9, 2013.

[104] S. Demyanenko, V. Nikul, S. Rodkin, A. Davletshin, M. B. Evgen'ev, and D. G. Garbuz, "Exogenous recombinant Hsp70 mediates neuroprotection after photothrombotic stroke," Cell Stress \& Chaperones, vol. 26, no. 1, pp. 103-114, 2021. 
[105] K. Nozaki, M. Nishimura, and N. Hashimoto, "Mitogen-activated protein kinases and cerebral ischemia," Molecular Neurobiology, vol. 23, no. 1, pp. 01-20, 2001.

[106] X. K. Tu, W. Z. Yang, S. S. Shi et al., "Baicalin inhibits TLR2/4 signaling pathway in rat brain following permanent cerebral ischemia," Inflammation, vol. 34, no. 5, pp. 463-470, 2011.

[107] X. Xue, X. J. Qu, Y. Yang et al., "Baicalin attenuates focal cerebral ischemic reperfusion injury through inhibition of nuclear factor $\kappa \mathrm{B}$ p65 activation," Biochemical and Biophysical Research Communications, vol. 403, no. 3-4, pp. 398404, 2010.

[108] X. K. Tu, W. Z. Yang, S. S. Shi, C. H. Wang, and C. M. Chen, "Neuroprotective effect of baicalin in a rat model of permanent focal cerebral ischemia," Neurochemical Research, vol. 34, no. 9, pp. 1626-1634, 2009.

[109] S. Yang, H. Wang, Y. Yang et al., "Baicalein administered in the subacute phase ameliorates ischemia-reperfusioninduced brain injury by reducing neuroinflammation and neuronal damage," Biomedicine \& Pharmacotherapy, vol. 117, article 109102, 2019.

[110] K. Yigitkanli, A. Pekcec, H. Karatas et al., "Inhibition of 12/15-lipoxygenase as therapeutic strategy to treat stroke," Annals of Neurology, vol. 73, no. 1, pp. 129-135, 2013.

[111] G. Jin, K. Arai, Y. Murata et al., "Protecting against cerebrovascular injury: contributions of 12/15-lipoxygenase to edema formation after transient focal ischemia," Stroke, vol. 39, no. 9, pp. 2538-2543, 2008.

[112] L. Cui, X. Zhang, R. Yang et al., "Baicalein is neuroprotective in rat MCAO model: role of 12/15-lipoxygenase, mitogenactivated protein kinase and cytosolic phospholipase A2," Pharmacology, Biochemistry, and Behavior, vol. 96, no. 4, pp. 469-475, 2010.

[113] S. Pallast, K. Arai, A. Pekcec et al., "Increased nuclear apoptosis-inducing factor after transient focal ischemia: a 12/15-lipoxygenase-dependent organelle damage pathway," Journal of Cerebral Blood Flow and Metabolism, vol. 30, no. 6, pp. 1157-1167, 2010.

[114] W. H. Li, Y. L. Yang, X. Cheng et al., "Baicalein attenuates caspase-independent cells death via inhibiting PARP-1 activation and AIF nuclear translocation in cerebral ischemia/reperfusion rats," Apoptosis, vol. 25, no. 5-6, pp. 354-369, 2020.

[115] D. Stephenson, K. Rash, B. Smalstig et al., "Cytosolic phospholipase A2 is induced in reactive glia following different forms of neurodegeneration," Glia, vol. 27, no. 2, pp. 110128, 1999.

[116] Y. W. Xu, L. Sun, H. Liang, G. M. Sun, and Y. Cheng, "12/15Lipoxygenase inhibitor baicalein suppresses PPAR $\gamma$ expression and nuclear translocation induced by cerebral ischemia/reperfusion," Brain Research, vol. 1307, pp. 149-157, 2010.

[117] H. Lee, Y. O. Kim, H. Kim et al., "Flavonoid wogonin from medicinal herb is neuroprotective by inhibiting inflammatory activation of microglia," The FASEB Journal, vol. 17, no. 13, pp. 1-21, 2003.

[118] J. Cho and H. K. Lee, "Wogonin inhibits ischemic brain injury in a rat model of permanent middle cerebral artery occlusion," Biological \& Pharmaceutical Bulletin, vol. 27, no. 10, pp. 1561-1564, 2004.

[119] Z. Kong, Q. Shen, J. Jiang, M. Deng, Z. Zhang, and G. Wang, "Wogonin improves functional neuroprotection for acute cerebral ischemia in rats by promoting angiogenesis via TGF- $\beta 1$," Annals of Translational Medicine, vol. 7, no. 22, pp. 639-639, 2019.

[120] P. A. Guerrero and J. H. McCarty, TGF- $\beta$ Activation and Signaling in Angiogenesis, IntechOpen, 2017.

[121] J. T. Qu, D. X. Zhang, F. Liu et al., "Vasodilatory effect of wogonin on the rat aorta and its mechanism study," Biological \& Pharmaceutical Bulletin, vol. 38, no. 12, pp. 1873-1878, 2015.

[122] J. H. Liu, H. Wann, M. M. Chen et al., "Baicalein significantly protects human retinal pigment epithelium cells against $\mathrm{H}_{2} \mathrm{O}_{2}$-Induced oxidative stress by scavenging reactive oxygen species and downregulating the expression of matrix metalloproteinase-9 and vascular endothelial growth factor," Journal of Ocular Pharmacology and Therapeutics, vol. 26, no. 5, pp. 421-429, 2010.

[123] H. M. Chao, M. J. Chuang, J. H. Liu et al., "Baicalein protects against retinal ischemia by antioxidation, antiapoptosis, downregulation of HIF- $1 \alpha$, VEGF, and MMP-9 and upregulation of HO-1," Journal of Ocular Pharmacology and Therapeutics, vol. 29, no. 6, pp. 539-549, 2013.

[124] N. Nakamura, S. Hayasaka, X. Y. Zhang et al., "Effects of baicalin, baicalein, and wogonin on interleukin-6 and interleukin-8 expression, and nuclear factor- $\kappa \mathrm{b}$ binding activities induced by interleukin- $1 \beta$ in human retinal pigment epithelial cell line," Experimental Eye Research, vol. 77, no. 2, pp. 195-202, 2003.

[125] C. Chen, D. Guo, and G. Lu, "Wogonin protects human retinal pigment epithelium cells from LPS-induced barrier dysfunction and inflammatory responses by regulating the TLR4/NF- $\kappa \mathrm{B}$ signaling pathway," Molecular Medicine Reports, vol. 15, no. 4, pp. 2289-2295, 2017.

[126] T. Yoshikawa, N. Ogata, H. Izuta, M. Shimazawa, H. Hara, and K. Takahashi, "Increased expression of tight junctions in ARPE-19 cells under endoplasmic reticulum stress," Current Eye Research, vol. 36, no. 12, pp. 1153-1163, 2011.

[127] C. Dai, S. Jiang, C. Chu, M. Xin, X. Song, and B. Zhao, "Baicalin protects human retinal pigment epithelial cell lines against high glucose-induced cell injury by up-regulation of microRNA-145," Experimental and Molecular Pathology, vol. 106, pp. 123-130, 2019.

[128] S. H. Jung, K. D. Kang, D. Ji et al., “The flavonoid baicalin counteracts ischemic and oxidative insults to retinal cells and lipid peroxidation to brain membranes," Neurochemistry International, vol. 53, no. 6-8, pp. 325-337, 2008.

[129] D. Kaur, V. Sharma, and R. Deshmukh, “Activation of microglia and astrocytes: a roadway to neuroinflammation and Alzheimer's disease," Inflammopharmacology, vol. 27, no. 4, pp. 663-677, 2019.

[130] L. P. Yang, H. L. Sun, L. M. Wu et al., "Baicalein reduces inflammatory process in a rodent model of diabetic retinopathy," Investigative Ophthalmology \& Visual Science, vol. 50, no. 5, pp. 2319-2327, 2009.

[131] X. J. Wang, C. S. Liu, and Z. X. Li, "The effect of baicalin on tissue aldose reductase activity and retinal apoptosis in diabetic rats," Chinese Journal of Diabetes, vol. 16, no. 8, p. 26, 2008.

[132] A. Othman, S. Ahmad, S. Megyerdi et al., "12/15-Lipoxygenase-derived lipid metabolites induce retinal endothelial cell barrier dysfunction: contribution of NADPH oxidase," PLoS One, vol. 8, no. 2, article e57254, 2013. 
[133] B. Uttara, A. Singh, P. Zamboni, and R. Mahajan, "Oxidative stress and neurodegenerative diseases: a review of upstream and downstream antioxidant therapeutic options," Current Neuropharmacology, vol. 7, no. 1, pp. 65-74, 2009.

[134] K. van Leyen, T. R. Holman, and D. J. Maloney, "The potential of 12/15-lipoxygenase inhibitors in stroke therapy," Future Medicinal Chemistry, vol. 6, no. 17, pp. 1853-1855, 2014.

[135] Y. Liu, Y. Zheng, H. Karatas et al., "12/15-lipoxygenase inhibition or knockout reduces warfarin-associated hemorrhagic transformation after experimental stroke," Stroke, vol. 48, no. 2, pp. 445-451, 2017.

[136] H. Karatas and C. Cakir-Aktas, "12/15 lipoxygenase as a therapeutic target in brain disorders," Noro Psikiyatri Arsivi, vol. 56, no. 4, pp. 288-291, 2019.

[137] Y. Tang, Z. Xiao, L. Pan et al., "Therapeutic targeting of retinal immune microenvironment with CSF-1 receptor antibody promotes visual function recovery after ischemic optic neuropathy," Frontiers in Immunology, vol. 11, article 585918, 2020.

[138] N. Hanioka, T. Isobe, T. Tanaka-Kagawa, and S. Ohkawara, "Wogonin glucuronidation in liver and intestinal microsomes of humans, monkeys, dogs, rats, and mice," Xenobiotica, vol. 50, no. 8, pp. 906-912, 2020. 